\title{
Making history accessible to people with visual impairments through interactive tangible 3D models
}

\author{
Jan BRUS ${ }^{\mathrm{a},{ }^{*}}$ Richard LAZNA ${ }^{\mathrm{a}}$ Radek BARVIR $^{\mathrm{a}}$ Alena VONDRAKOVA ${ }^{\mathrm{a}}$ \\ a Palacký University Olomouc, Department of Geoinformatics, radek.barvir@upol.cz, jan.brus@upol.cz, alena.vondrakova@upol.cz \\ * Corresponding author
}

Keywords: 3D modelling, 3D printing, tangible, visual impairment, architecture

\section{Abstract:}

In the case of presenting historical objects to the public, 3D models play a crucial role in stimulating the imagination and education. However, these models are supplemented with additional textual information or placed under glass very often. Most of the human population can still perceive the models and use additional text or infographics. However, there is a significant group of people with visual impairments for whom a comprehensive interpretation of the model without the ability to touch and access the contextual information is an impossible task. Therefore, there are already approaches that aim to make various models, which can also be used in museum exhibitions, accessible to people with visual impairments. One of the revolutionary technologies that have made the production of touch models easy is additive manufacturing. This technology, more generally known as 3D printing, allows the creation of diverse objects. The limitation, in this case, is perhaps only the degree of creativity and, to some extent, the specific technical characteristics of 3D printers and the material used for printing. 3D printing allows the creation of replicas of historical objects (Fig. 1) . However, 3D models are implicitly static and do not respond in any way to any touch. In the case of historical objects, it is very common to get contextual information related to the part of the object and the spatial representation. Current 3D printing capabilities already make using advanced materials and creating interactive 3D models possible. As a result, models can be created in the way they introduce the user to the historical situation using the principles of interactivity.

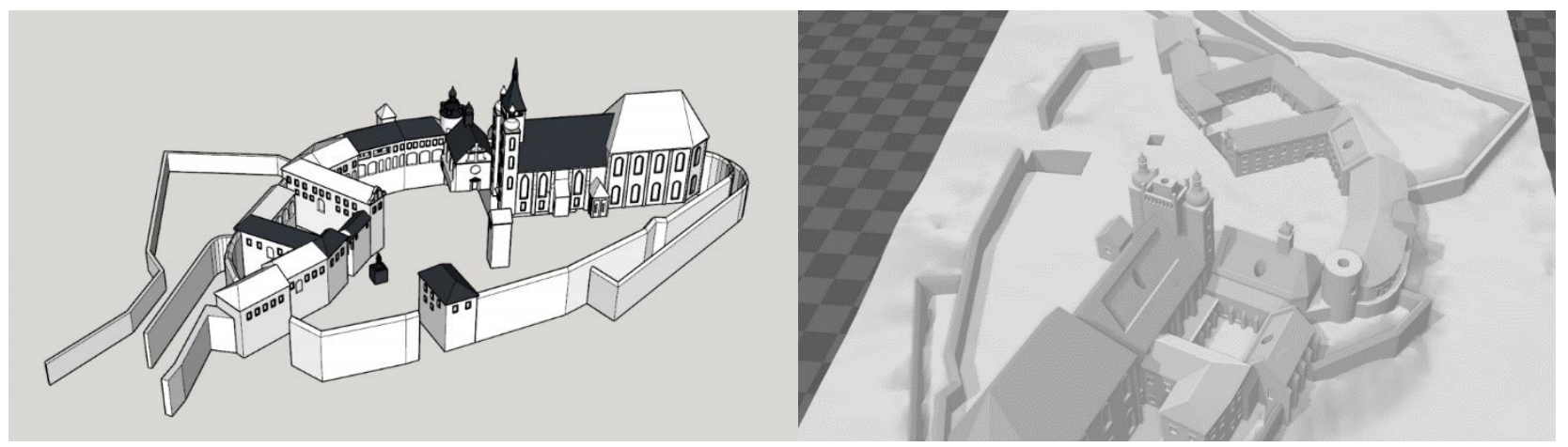

Figure 1. The 3D model of the St. Wenceslas Hill in Olomouc with black parts which will be tangible (left). The 3D model without conductive parts (right).

This paper aims to present a case study of an interactive haptic 3D model on the example of the model of St. Wenceslas Hill in Olomouc. This model is supplemented by an interface providing audio information for the people with severe visual impairment. For the creation of the model, the TouchIt3D technology developed at Palacký University Olomouc was used. This technology works on the principle of connecting a mobile device with a model printed on a $3 \mathrm{D}$ printer for interaction. The technology uses a combination of conductive and non-conductive plastic. The material used is polylactic acid (PLA), where PLA can be used in both conductive and non-conductive versions. The conductive part of the model responds to touch and serves as a signal transmitter. The conductive PLA contains carbon, which conducts the electrical impulse caused by the touch. The second essential component is the mobile device, on whose several areas act as virtual buttons (Fig. 2). Touch is transmitted to the screen, interaction with the control occurs, and thus augmenting information about the part of the model can be obtained through text-to-speech. The CraftBot FLOW IDEX XL printer was used to print the model itself, using two extruders for printing. It is the two extruders that give the ability to print the model from two materials simultaneously, in this case, conductive and non-conductive filament. Before the actual creation, it was necessary to solve the final scale of the model due to the design of the number of conductive elements and their positioning. After calculations, the scale was set to 1:1000. 


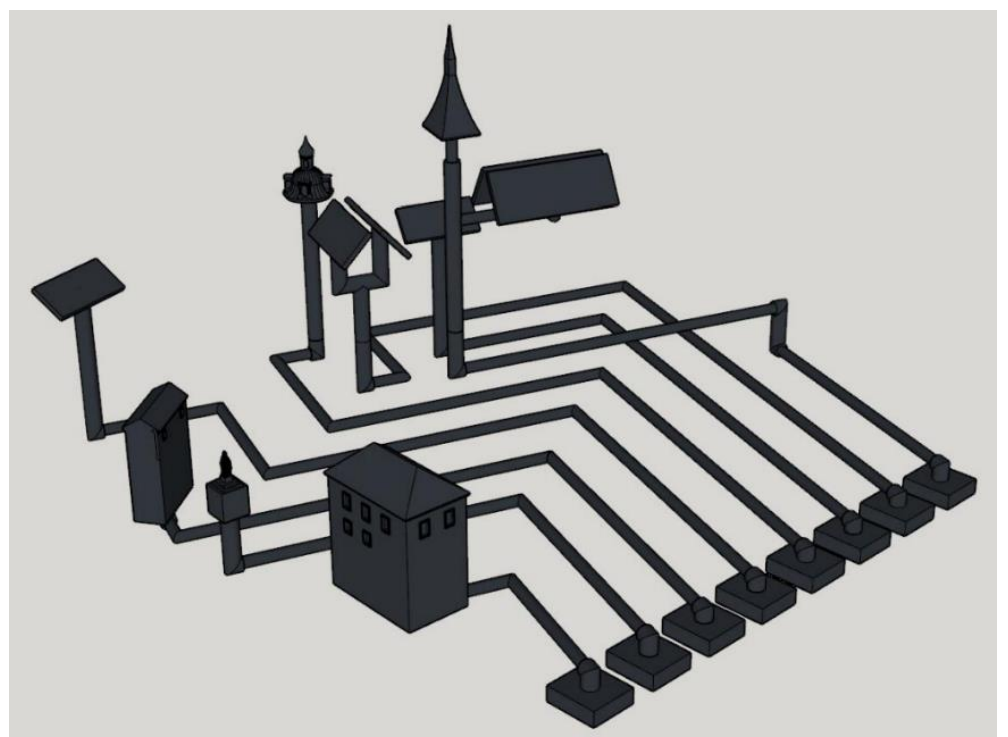

Figure 2. Conductive parts of the model with square conductive areas for contact with tablet.

The next step was to design modifications and simplify the model for people with severe visual impairment. The modifications consisted mainly of adjusting details and flattening some surfaces to make them more suitable for touch. Then came the actual creation of the conductive parts. After considering several aspects, the decision was made to create a total of eight interactive elements. In some cases, the entire model was chosen as the conductive part. In others, the selected part was modified by adding a touch surface, with the rule that the conductive part always exactly copied the non-conductive part. Each conductive element is composed of one or more touch surfaces modeled in such a way as to result in contact of the element with the tablet screen. The conductive elements are designed to be spatially nonintersecting and spaced apart sufficiently to prevent misinterpretation of the signal. After the conductive part of the model was created, the next step was to perform a logical difference operation so that in the result, there was a designed conductive part and then a non-conductive part, for which any parts that interfered with the conductive part were removed. In simple terms, the conductive part was left as it was modeled, and everything common to both parts was removed from the non-conductive part. The parts of the model thus divided were then joined in a slicer software and printed. The last part of the resulting model is the second part of the base, which was modeled to create a space for the tablet to be placed to prevent, at least to some extent, unwanted manipulation of the device and disabling functionality. The connection between the base and the main model, consisting of a conductive and a non-conductive part, is solved using magnets. An important result of the work, supporting the functionality of the main model, is the environment created to control the textual information for text into speech conversion. This environment can also display extended contextual information to the second user when needed. The final model is $30 \mathrm{~cm}$ long and is printed in white and black color (Fig. 3). It is a prototype that will be used as proof of concept and basement for further interactive models based on modeling UNESCO heritage objects in Czechia.

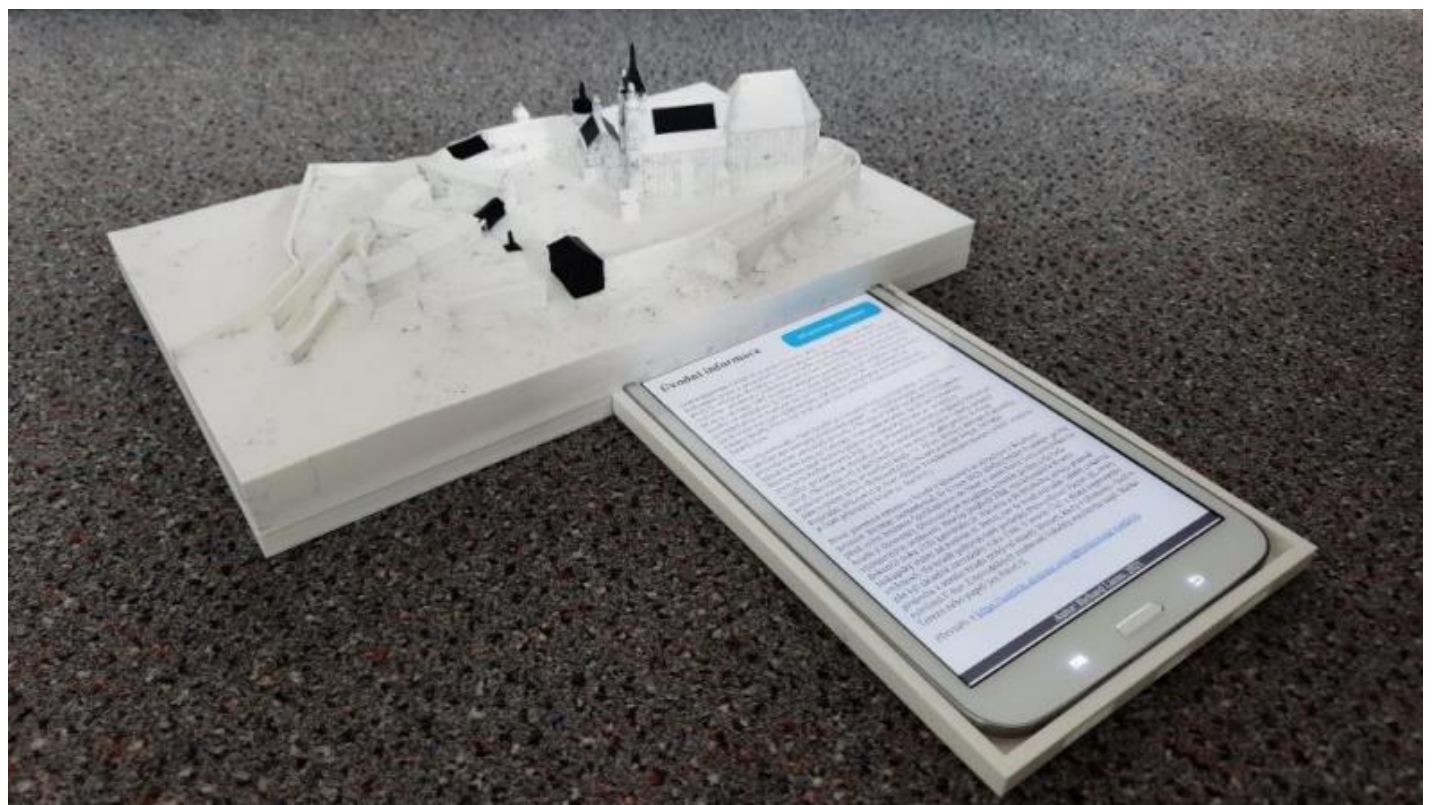

Figure 3. Final prototype with connected tablet and application for contextual information with text-to-speech function. 